\title{
The temporo-parietal junction contributes to global gestalt perception - evidence from studies in chess experts
}

\author{
Johannes Rennig ${ }^{1 *}$, Merim Bilalić ${ }^{2 \dagger}$, Elisabeth Huberle ${ }^{1,3}$, Hans-Otto Karnath ${ }^{1,4}$ and \\ Marc Himmelbach ${ }^{1}$ \\ 1 Division of Neuropsychology, Center of Neurology, Hertie-Institute for Clinical Brain Research, University of Tübingen, Tübingen, Germany \\ 2 Department of Neuroradiology, University of Tübingen, Tübingen, Germany \\ ${ }^{3}$ Neurology and Neurorehabilitation Center, Luzerner Kantonsspital, Luzern, Switzerland \\ ${ }^{4}$ Department of Psychology, University of South Carolina, Columbia, SC, USA
}

\section{Edited by:}

Magdalena Chechlacz, University of

Oxford, UK

\section{Reviewed by:}

Harriet A. Allen, University of

Birmingham, UK

Dario Cazzoli, University of

Oxford, UK

\section{*Correspondence:}

Johannes Rennig, Division of Neuropsychology, Center of

Neurology, Hertie-Institute for Clinical Brain Research, University of Tübingen, Hoppe-Seyler-Str. 3, D-72076, Tübingen, Germany e-mail: johannes.rennig@ uni-tuebingen.de

${ }^{\dagger}$ These authors have contributed equally to this work.
In a recent neuroimaging study the comparison of intact vs. disturbed perception of global gestalt indicated a significant role of the temporo-parietal junction (TPJ) in the intact perception of global gestalt (Huberle and Karnath, 2012). This location corresponded well with the areas known to be damaged or impaired in patients with simultanagnosia after stroke or due to neurodegenerative diseases. It was concluded that the TPJ plays an important role in the integration of individual items to a holistic percept. Thus, increased BOLD signals should be found in this region whenever a task calls for the integration of multiple visual items. Behavioral experiments in chess experts suggested that their superior skills in comparison to chess novices are partly based on fast holistic processing of chess positions with multiple pieces. We thus analyzed BOLD data from four fMRI studies that compared chess experts with chess novices during the presentation of complex chess-related visual stimuli (Bilalić et al., 2010, 2011a,b, 2012). Three regions of interests were defined by significant TPJ clusters in the abovementioned study of global gestalt perception (Huberle and Karnath, 2012) and BOLD signal amplitudes in these regions were compared between chess experts and novices. These cross-paradigm ROI analyses revealed higher signals at the TPJ in chess experts in comparison to novices during presentations of complex chess positions. This difference was consistent across the different tasks in five independent experiments. Our results confirm the assumption that the TPJ region identified in previous work on global gestalt perception plays an important role in the processing of complex visual stimulus configurations.

Keywords: gestalt perception, visual grouping, temporo-parietal junction, object perception, expertise, fMRI, simultanagnosia, chess

\section{INTRODUCTION}

A crucial aspect of visual object recognition is the grouping of single elements to a global entity or so-called gestalt (Wertheimer, 1923; Koffka, 1935). The neuronal correlates of global processing or visual integration are still a matter of lively debates. Patients suffering from simultanagnosia, the inability to perceive a global gestalt first described as part of the Bálint syndrome (Bálint, 1909), typically show bilateral lesions in posterior parieto-temporal brain areas, whereas a remarkable variability concerning the exact localization is still prevalent (Rizzo and Hurtig, 1987; Friedman-Hill et al., 1995; Rafal, 1997; Karnath et al., 2000; Tang-Wai et al., 2004; Valenza et al., 2004; Huberle and Karnath, 2006, 2010; Thomas et al., 2012). Moreover, there is an inconsistency between functional imaging studies that attributed global perception to unilateral regions along the ventral visual pathway (Fink et al., 1996, 1997a,b) and other studies that found an association with posterior parietal and/or parieto-temporal areas (Yamaguchi et al., 2000; Himmelbach et al., 2009; Huberle and Karnath, 2012; Zaretskaya et al., 2013).
Research in chess experts provided a large body of data addressing neuronal correlates of visual skills (Bilalić et al., 2010, 2011a,b, 2012; Krawczyk et al., 2011). For research on object recognition and visual integration chess appears to be particularly suitable as it features various, clearly distinguishable individual objects that allow the composition of complex stimulus configurations with graded complexity. Furthermore, chess provides the opportunity to compare highly trained experts with novices based on a standardized rating system (Elo, 1978). Behavioral studies demonstrate that domain-specific knowledge, acquired through prolonged and focused training (Ericsson et al., 1993), enables experts to quickly grasp the essence of complex chess positions (DeGroot, 1978; Bilalić et al., 2008a). Instead of perceiving individual chess objects serially like novices, experts perceive meaningful units of several objects, called chunks (Chase and Simon, 1973) or templates (Gobet and Simon, 1996), which are linked with typical actions through pattern recognition mechanisms (Bilalić et al., 2008a,b, 2009, 2010). A typical chess position featuring numerous individual objects represents a single meaningful unit to chess experts. In a recent series of fMRI 
studies, Bilalić et al. (2010, 2011a,b, 2012) demonstrated that chess experts also showed different neuronal response patterns in the ventral visual system compared to novices. Typically, chess experts showed higher signal increases mostly in the temporal lobe compared to novices during the observation of chess stimuli. A study by Krawczyk et al. (2011) using comparable stimulus material revealed a similar result pattern with higher signals in temporal and frontal brain areas for experts compared to novices.

Based on the assumption that the behavioral advantage of chess experts is, at least partially, based on superior skills in the visual integration of multiple chess pieces we hypothesized that there should be a difference in the BOLD signal in regions that were functionally mapped in an independent study of global perception using substantially different stimulus material (Huberle and Karnath, 2012). In detail, the temporo-parietal junction (TPJ) was investigated by using an independent set of data from chess experts as well as novices. Several studies investigating neuronal processes of visual grouping used stimuli that may have evoked neuronal responses depending on low-level visual features like spatial frequencies of luminance changes (e.g. Fink et al., 1996; Huberle and Karnath, 2012). The stimuli examined in the ROI analyses of the present approach were substantially different from simple hierarchical Navon-like (Navon, 1977) stimulus material. The relationships between chess pieces that support the emergence of a global percept are not based on low-level visual features but on the knowledge about these pieces and their semantic relations. We compared signal levels in chess experts and novices in region of interest (ROI) analyses using four independent fMRI datasets taken from previously published studies on chess expertise (Bilalić et al., 2010, 2011a,b, 2012). We analyzed three ROIs defined by the data from Huberle and Karnath (2012). All three regions were located in the area of the right or left TPJ. While in three of these studies (Bilalić et al., 2010, 2011b, 2012) visual processing required an analysis of highly complex chess positions, one task (Bilalić et al., 2011a) focused on simple object perception.

\section{MATERIALS AND METHODS PARTICIPANTS}

Eleven subjects ( 3 males/ 8 females; mean age 24.6 years, SD \pm 0.7 years) participated in the study of Huberle and Karnath (2012). Subjects had normal or corrected to normal vision and reported no history of neurological impairment affecting their visual capacity. In all four studies of Bilalić et al. (2010, 2011a,b, 2012) expert as well as novice chess players participated (Table 1). Tournament players get rated based on their performance against other rated players. The international chess Elo scale is an interval scale with a theoretical mean of 1500 and standard deviation of 200 (Elo, 1978). Experts are players with a rating of 2000 Elo points or more. The experts included in the present studies were rated with an average around 2100 points. Novice players were hobby players who played chess occasionally. Their chess skills were clearly inferior to experts but they had no difficulties in identifying chess pieces and their functions. All players were male and right-handed. The Institutional Review Board of the Ethic Committee of Tübingen University approved both studies and
Table 1 | Participants in the studies of Bilalić et al. (2010, 2011a,b, 2012): group, mean age, mean skill level as measured by the Elo rating (see Methods) with standard deviation (SD), number of standard deviations above the mean, and number of participants in each group in all four experiments.

\begin{tabular}{llllll}
\hline Experiment & Group & Age \pm SD & Elo \pm SD & $\begin{array}{l}\text { SDs above } \\
\text { mean }\end{array}$ & $\boldsymbol{n}$ \\
\hline 1 & & & & 3 & 7 \\
& Expert & $30 \pm 2$ & $2117 \pm 53$ & - & 8 \\
\hline Novice & $28 \pm 1$ & - & 3 & 8 \\
\hline 3 & Expert & $29 \pm 7$ & $2130 \pm 147$ & 3 \\
\hline & Novice & $29 \pm 5$ & - & - & 7 \\
\hline 4 & Expert & $30 \pm 2$ & $2117 \pm 53$ & 3 & 8 \\
\hline & Novice & $29 \pm 1$ & - & - & 8 \\
& Expert & $30 \pm 5$ & $2108 \pm 148$ & 3 & 15
\end{tabular}

written informed consent was obtained from all participants. All studies were performed in accordance with the ethical standards laid down in the 1964 Declaration of Helsinki.

\section{PROCEDURE AND STIMULI}

In the study of Huberle and Karnath (2012) a global circle or square was constructed from smaller local images of circles or squares. Figure 1A illustrates examples from this set of stimuli. Objects at the global level were scrambled by exchanging a certain percentage of the local images with each other, thereby producing a set of stimuli at scrambling levels of $20-, 40-, 60-$ and $80 \%$. The behavioral results of a two-alternative forced choice (2AFC) task to report the category of the object at the global level ("global circle" vs. "global square") showed that in $20 \%$ scrambled stimuli the global gestalt was easily perceived (97\% Correct) whereas $80 \%$-scrambling almost completely prevented global perception (52\% Correct).

The four studies of Bilalić et al. (2010, 2011a,b, 2012) comprised five analyses of complex chess related visual stimuli. We combined the data from two analyses, originally reported by Bilalić et al. (2010, 2012), because the data of these analyses came from the same imaging sessions with the same subjects. In the following we address this set of data as Experiment 4.

\section{Experiment 1 (Bilalić et al., 2011b)}

Participants indicated if the current stimulus was the same as the previous one. There were four classes of stimuli: chess and face stimuli, which were presented upright or upside-down (Figure 1B). The face stimuli were black-white pictures of students (Leube et al., 2001, 2003). The chess stimuli were fullboard positions taken from a four-million-chess-game database (ChessBase Mega Base 2007, ChessBase GmbH, Hamburg, Germany; www.chessbase.com). Stimuli from four categories (faces upright, chess upright, faces inverted, chess inverted) were presented in blocks of five stimuli. A single stimulus lasted for $2.75 \mathrm{~s}$ and was followed by a random noise mask for $0.25 \mathrm{~s}$. A baseline (gray screen with a central fixation cross) was presented at the beginning, after each block, and at the end of the experiment for 


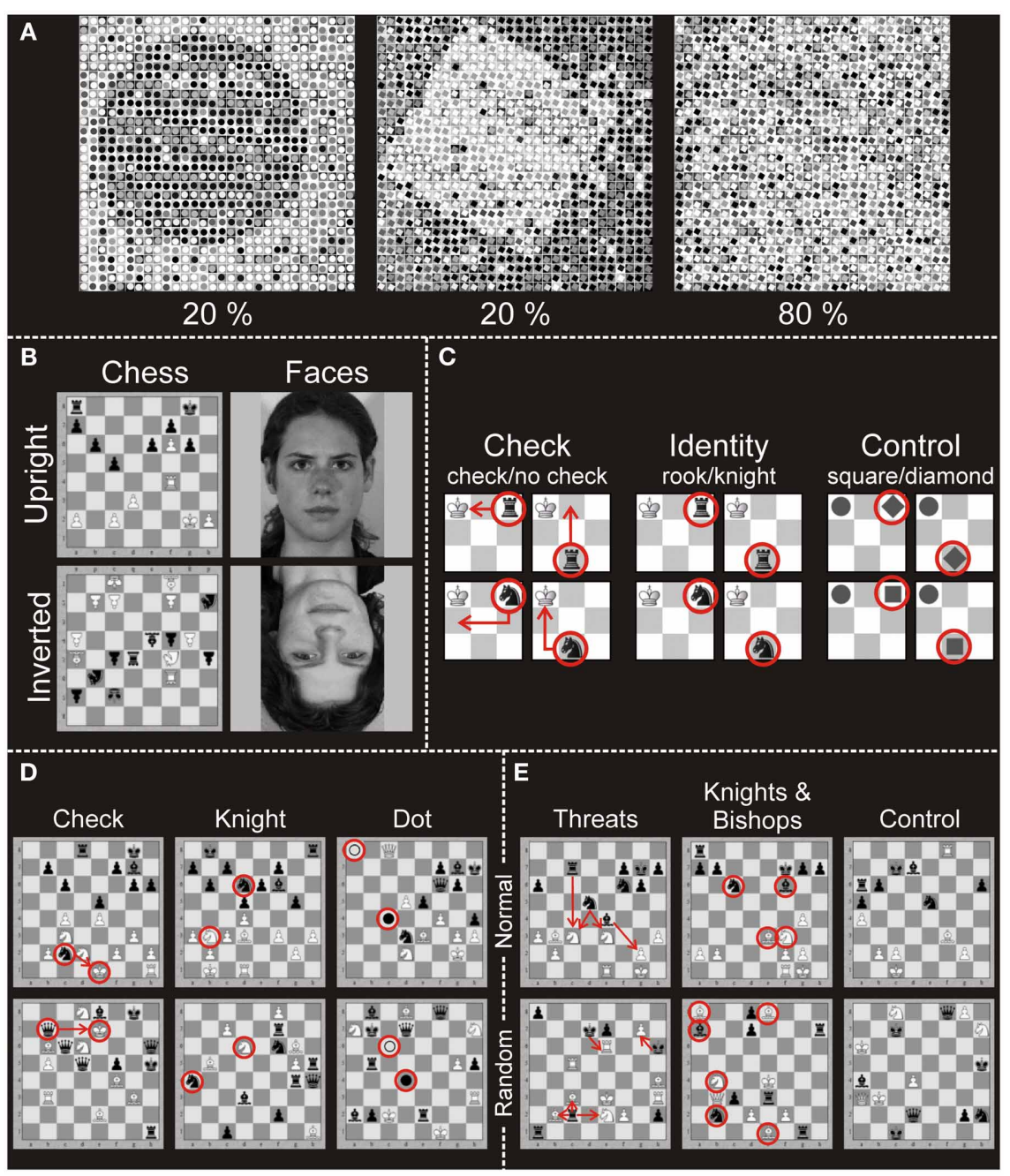

FIGURE 1 | Stimuli applied in the different experiments. (A) Illustration of the global stimuli used in the experiment of Huberle and Karnath (2012). The pictures show two $20 \%$ scrambled global objects (circle, square; intact global perception) and one $80 \%$ scrambled global object (disturbed global

perception). (B) Stimuli used in Experiment 1 (Bilalić et al., 2011b). Pictures of full-board chess positions or faces were presented upright or inverted. Participants had to indicate whether the currently presented stimulus matched the previously presented stimulus. (C) Stimuli of Experiment 2 (Bilalic et al., 2011a). The stimuli were presented on a $3 \times 3$ miniature chess board. In the check task participants indicated whether the black piece (knight or rook) gives the white king check. In the identity task participants indicated whether the presented black piece is a rook or a knight. In the control task participants identified geometrical shapes (square or diamond). (D) Stimuli of
Experiment 3 (Bilalić et al., 2011b). Pictures of full-board chess positions were presented. Participants had to indicate whether the white king was in check in the check task, whether there were knights of both colors presented in the knight task, or whether two dots (black and white) were present in the control (dot) task. (E) Stimuli of Experiment 4 (Bilalić et al., 2010, 2012). Pictures of full-board chess positions were presented. Participants had to indicate whether the number of black threats (how many times black can take white) was four in the threat condition, whether the number of knights and bishops was four in the knights and bishops task and whether the number of all pieces on the board was 15 in the control task. In all three tasks of Experiments 3 and 4, there were two types of positions: normal (taken from chess games of masters) and random (pieces were randomly distributed on the board).
14 s. All four conditions were presented four times in each of three runs (12 blocks of each condition in all runs).

\section{Experiment 2 (Bilalić et al., 2011a)}

This experiment featured three tasks. In the check task, participants indicated if the white king was attacked (i.e., given check) by the only present black piece. There were four different stimuli with two pieces on a $3 \times 3$ miniature chess board (Figure 1C). The white king was always on the first square of the upper left corner, while the identity of the other piece (knight or rook) and its location (middle of the lower row or the end of the upper row) varied. In the Identity task, participants were presented with the same stimuli as in the check task, but this time they identified the black piece presented. In the non-chess control task, chess pieces had been exchanged by gray-colored geometrical shapes (a circle for the king; a diamond and square for knight and rook, respectively). In parallel to the two chess tasks, the identity (diamond or square) and position (middle of the lower row or the end of the upper row) of the target stimulus were varied, and participants indicated its shape. Stimuli were presented in 
a block design. There were four runs and 12 blocks in each of them (four blocks for each condition in a single run). The runs were block-randomized and counterbalanced across participants. The experiment started with an empty $3 \times 3$ board (baseline) for $13.5 \mathrm{~s}$ and was followed by a written instruction for $3 \mathrm{~s}$ indicating the task type (check, identity, or control). After the instruction an empty $3 \times 3$ board was presented for $1.5 \mathrm{~s}$. After $1 \mathrm{~s}$ a black center cross appeared and was presented for $0.5 \mathrm{~s}$ to warn participants about the upcoming stimulus. The following stimulus lasted for 2 s. There were four trials (stimuli) in a block, and after each block the baseline was presented.

\section{Experiment 3 (Bilalić et al., 2011b)}

These tasks were similar to the previous experiment-recognizing if the white king was in check (check task), recognizing if knights of either color were present (knight task), and recognizing if a dot of either color was presented (dot task-see Figure 1D). The stimuli, however, consisted of full chess positions (containing 15-18 pieces) presented on a full $8 \times 8$ square chess board. There were two types of positions-normal and random. The normal positions were taken from the same ChessBase database as in Experiment 1 and were typical middle-game positions of master games not previously known to the participants. The random positions were generated by distributing the pieces on the board randomly using the rule that any piece of either color can occur on any square (Vicente and Wang, 1998; Gobet and Waters, 2003). There were four runs with 12 blocks each, comprising two blocks per condition ( 3 tasks $\times 2$ position types) in a single run. The runs were block-randomized and counterbalanced across participants. The experiment started with a gray screen with a black center cross, which lasted 5-10 s, immediately followed by the instruction for $2.5 \mathrm{~s}$, after which the actual block started. The stimulus was presented for $4 \mathrm{~s}$ and was followed by a mask made of a scrambled chess position, which lasted for $0.5 \mathrm{~s}$. There were four trials (stimuli) in each block, and baseline was always presented after each block.

\section{Experiment 4: (Bilalić et al., 2010, 2012)}

In this experiment full chess boards with 15-18 pieces were presented in normal and random positions. New middle-game positions were sampled from the ChessBase database. The tasks involved enumerations of chess pieces and their relations (Figure 1E). In the threats task, players indicated whether the number of threats (black to white) was four. In the knights and bishops condition, the task was to indicate whether the number of knights and bishops of both colors was four. Finally, in the non-chess control task, all pieces regardless of color or type were counted (indicate if the number is 15).

There were six runs, two for each task. There was only one task (e.g., threats task) in a single run. In one run, 10 meaningful and 10 meaningless stimuli were presented randomly. The runs were block-randomized and counterbalanced across participants. We first presented a starting board (all pieces at their initial location) with a fixation cross as a baseline with jittered duration (6-10 s). After a short gap $(0.5 \mathrm{~s})$, the target stimulus was presented until response, followed by the baseline of the next trial. Before the actual sessions, participants were given two practice trials for each task. The reaction time (i.e., the time to complete the task) was the time between stimulus onset until the participant pressed the button.

In all experiments, the stimuli were projected on a screen above the head of the participant via a video projector placed in the adjacent room. The setup resulted in a visual field of $14.6^{\circ}$ for the whole scene. Participants saw the stimuli through a mirror mounted on the head coil and indicated their decision by pressing one of two buttons of an MRI-compatible response device held in their right hand.

\section{MRI ACQUISITION}

All fMRI data were acquired using a 3-T scanner (Siemens Trio) with a 12-channel head coil at the University Hospital of Tübingen. All measurements covered the whole brain using standard echo-planar-imaging (EPI) sequences. For the experiments of Bilalic et al. (2010, 2011a,b, 2012) the following parameters were used: $\mathrm{TR}=2.5 \mathrm{~s} ; \mathrm{FOV}=192 \times 192 \mathrm{~mm}$; TE $=35 \mathrm{~ms}$; flip angle: $90^{\circ}$; matrix size $=64 \times 64 ; 36$ slices with thickness of $3.2+0.8 \mathrm{~mm}$ gap resulting in voxels with a resolution of $3 \times 3 \times$ $4 \mathrm{~mm}^{3}$. The study of Huberle and Karnath (2012) used the following parameters: $\mathrm{TR}=2 \mathrm{~s} ; \mathrm{FOV}=192 \times 192 \mathrm{~mm}$; TE $=40 \mathrm{~ms}$; flip angle: $90^{\circ}$; matrix size $=64 \times 64 ; 24$ axial slices with a thickness of $5 \mathrm{~mm}$.

\section{FUNCTIONAL MRI DATA ANALYSIS}

The imaging data of Huberle and Karnath (2012) were originally processed using Brain Voyager ${ }^{\circledR}$, whereas the data of Bilalic et al. (2010, 2011a,b, 2012) were analyzed using SPM5 (Wellcome Department of Imaging Neuroscience, London, UK; http://www. fil.ion.ucl.ac.uk/spm). Brain Voyager ${ }^{\circledR}$ and SPM differ from each other in some crucial aspects. For example, volume normalization in BrainVoyager ${ }^{\circledR}$ transforms the data to the Talairach space whereas normalization in SPM is based on templates in MNI space (Goebel et al., 2006; Lancaster et al., 2007). For the sake of a direct transfer between the studies we re-analyzed the dataset of Huberle and Karnath (2012) using SPM8 (Wellcome Department of Imaging Neuroscience, London, UK; http://www. fil.ion.ucl.ac.uk/spm). Only for the data of Huberle and Karnath (2012) temporal offsets of slice acquisition were accounted for by a temporal realignment. For both studies further preprocessing included spatial realignment of all images of a subject to the mean functional image for motion correction. Only in the studies by Bilalić et al. (2010, 2011a,b, 2012) residual motion artifacts induced by a susceptibility-by-movement interaction were additionally addressed using the unwarp function of SPM5. The mean EPI and all functional images were co-registered to the anatomical image for every participant. All images were normalized using the respective T1-weighted template and smoothed with a FWHM of $8 \mathrm{~mm}$. Modeling of the time series of hemodynamic activation was based on the canonical response function as implemented in SPM5 and SPM8. A high-pass filter with a cutoff of $128 \mathrm{~Hz}$ eliminated low-frequency noise components and a correction for temporal autocorrelation in the data was applied using an autoregressive $\mathrm{AR}(1)$ process.

In the re-analysis of the Huberle and Karnath (2012) data, predictors for each experimental condition were constructed by 
a convolution of stimulus onsets for 20-, 40-, 60- and 80\%scrambled objects with the hemodynamic response function. The resulting design matrices comprised 4 experimental regressors, one for each scrambling level. Additionally, we included six covariates to capture residual movement-related artefacts. We used the individual participants' contrast images obtained from the first-level analysis for the second-level analysis. Areas involved in the intact perception of global gestalt were identified as those voxels that showed significantly higher signals for $20 \%$-scrambled objects (intact global perception) compared to $80 \%$-scrambled objects (disturbed global perception) based on a voxel-level threshold of $p<0.001$ (uncorr.) with a cluster extent of at least 50 voxels in the area of the TPJ. We used a relatively liberal threshold to get bilateral ROIs and extend the analysis also to a left-sided TPJ area that was delineated using the same methods and thresholds that served for the right hemisphere. The results of this re-analysis were topographically consistent with the original results produced with Brain Voyager ${ }^{\circledR}$. However, because of small differences in the statistical procedures between both analysis packages the extent of individual clusters based on individual thresholds were slightly different. The individual clusters that resulted from the re-analysis and which were used for the later ROI analyses are specified in Figure 2. In the further analyses we will label the three ROIs according to their localization as right, left anterior and left posterior TPJ ROI.

In the first two experiments of Bilalić and colleagues (Bilalić et al., 2011a,b), all trials were modeled with their full duration. In Experiment 3 (Bilalić et al., 2011b) the first second and in Experiment 4 (Bilalić et al., 2010, 2012) the first three seconds of each trial were used in order to keep the duration for each condition constant. The rest of the trial was also explicitly specified as a nuisance regressor, while the baseline was implicitly modeled. The mean percent signal changes (PSC) within each ROI were extracted for each participant and condition using MarsBar (http://marsbar.sourceforge.net). The PSC was calculated by dividing the maximum of the time course of the respective estimated event for this condition by the beta value for the constant session mean regressor. PSC values from experts and novices for the respective experiments and conditions were then analyzed with repeated measures ANOVAs.

\section{RESULTS}

In all experiments, chess experts showed a clear behavioral advantage compared to novices for chess related stimuli but not for the control stimuli (for details see: Bilalić et al., 2010, 2011a,b, 2012). To have an overview over all experiments and the respective results see Table 2 .

\section{EXPERIMENT 1}

For Experiment 1 we calculated a $2 \times 2 \times 2$ repeated measures ANOVA with the following factors and levels: expertise (expert vs. novice) $\times$ task (chess vs. face) $\times$ presentation (normal vs. inverted presentation).

\section{Right TPJ}

Experts showed stronger activation in the right TPJ area compared to novices depending on the stimulus category administered in the particular tasks (Figure 3A). Significantly stronger activations were evident for chess related stimuli in experts, while we found no significant difference between experts and

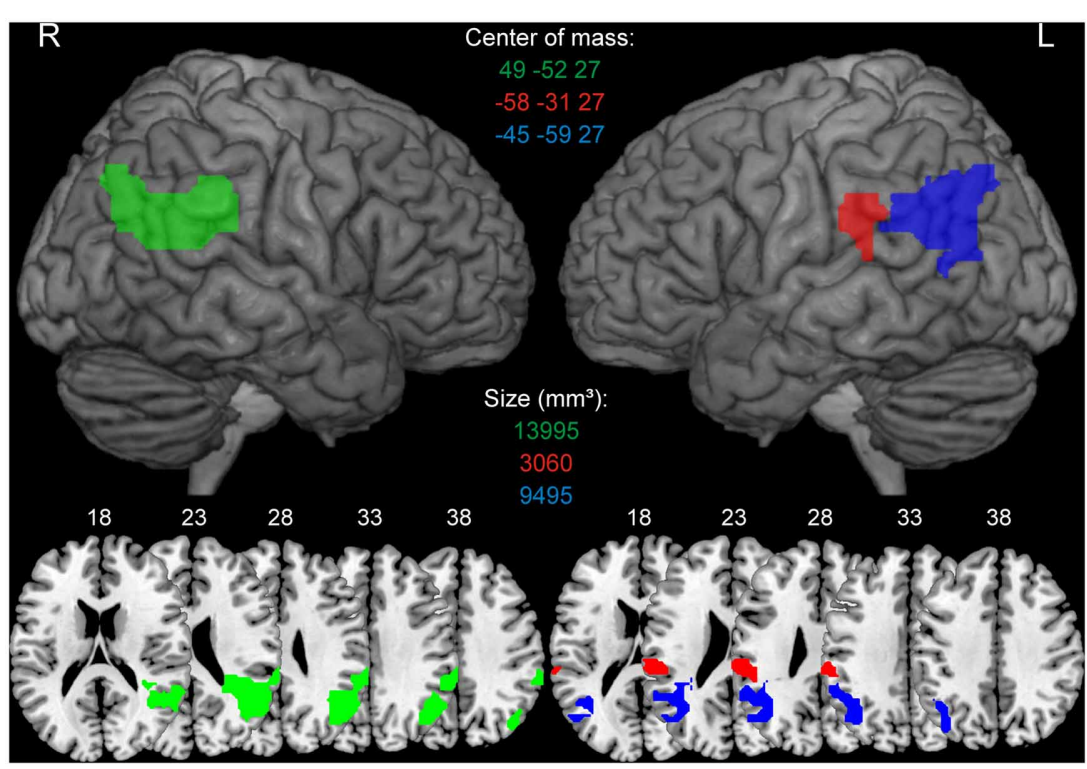

FIGURE 2 | Regions of interests (ROIs) derived from Huberle and Karnath (2012). ROls were identified as those voxels that showed significantly higher BOLD signals for $20 \%$-scrambled objects (intact global perception) compared to $80 \%$-scrambled objects (disturbed global perception) based on a voxel-level threshold of $p<0.001$ (uncorr.). The green color indicates the right TPJ ROI, red describes the left anterior TPJ $\mathrm{ROI}$, blue indicates the left posterior TPJ ROI. ROls are presented on a 3D rendered surface and axial slices for the left and right hemisphere. $\mathrm{MNI}$ coordinates of the center of mass and size of every $\mathrm{ROI}$ in $\mathrm{mm}^{3}$ is denoted in the corresponding color. 
Table 2 | Results of statistical comparisons between experts and novices are indicated by a ' + ' if a significant difference for the respective experiment and factor combination was observed and ' 0 ' if the difference was not significant.

\begin{tabular}{|c|c|c|c|c|c|c|c|c|c|c|c|}
\hline \multirow{2}{*}{$\begin{array}{l}\text { Experiment } \\
\text { task }\end{array}$} & \multicolumn{2}{|c|}{$1 \mathrm{~N}-$-Back } & \multicolumn{3}{|c|}{$\begin{array}{c}2 \text { Detection } \\
\text { (mini chess board) }\end{array}$} & \multicolumn{3}{|c|}{$\begin{array}{c}3 \text { Detection } \\
\text { (full chess board) }\end{array}$} & \multicolumn{3}{|c|}{4 Counting } \\
\hline & Chess & Faces & $\begin{array}{l}\text { Check/ } \\
\text { no check }\end{array}$ & $\begin{array}{l}\text { Rook/ } \\
\text { knight }\end{array}$ & Control & $\begin{array}{l}\text { Check/ } \\
\text { no check }\end{array}$ & Identity & Control & Threats & $\begin{array}{l}\text { Knights \& } \\
\text { bishop }\end{array}$ & Control \\
\hline \multicolumn{12}{|l|}{ TPJ RIGHT } \\
\hline Result & + & 0 & 0 & 0 & 0 & + & + & + & + & + & 0 \\
\hline \multicolumn{12}{|c|}{ TPJ LEFT ANTERIOR } \\
\hline \multicolumn{12}{|c|}{ TPJ LEFT POSTERIOR } \\
\hline Result & + & 0 & 0 & 0 & 0 & $+^{*}$ & 0 & 0 & 0 & 0 & 0 \\
\hline
\end{tabular}

The asterisks mark significant results derived from preceding full factorial analyses with p-values slightly above 0.05 (please see results section for Experiment 3).

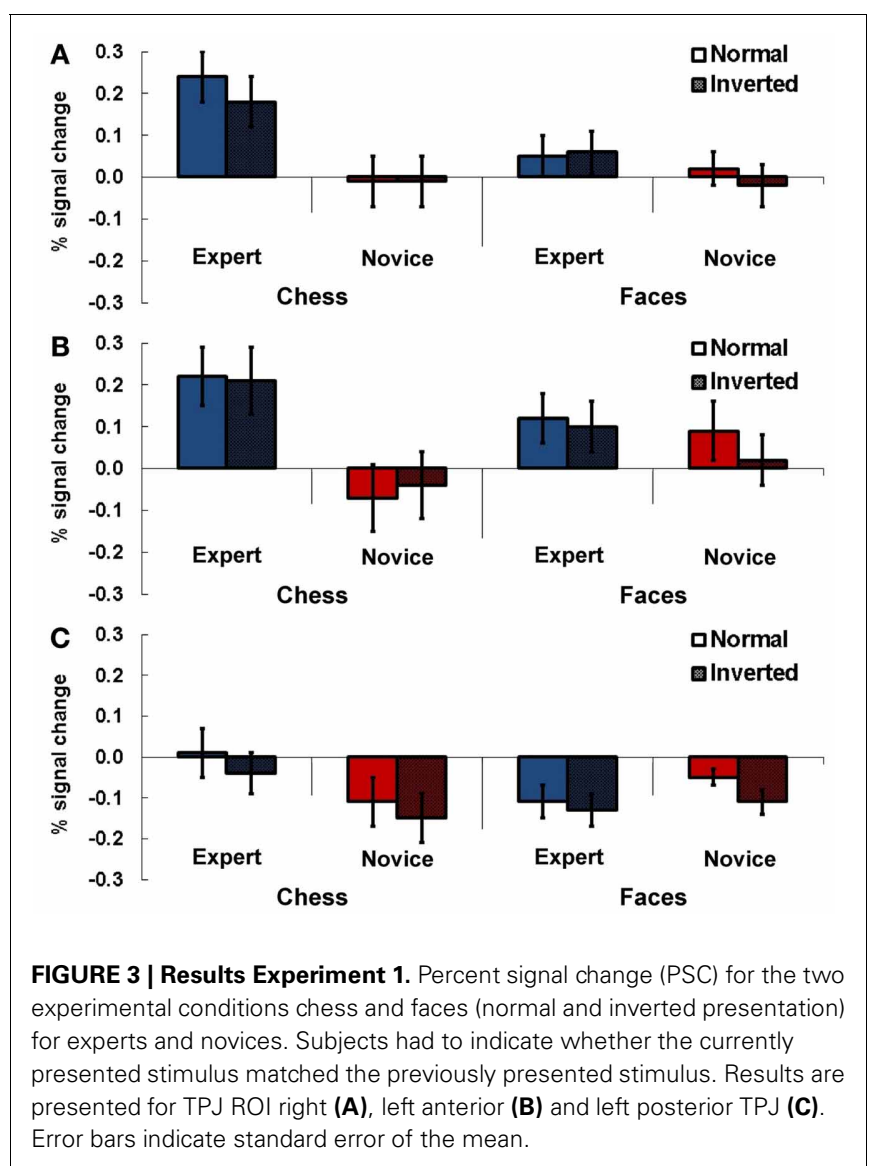

novices for faces. The statistical analysis showed a significant main effect for task $\left[F_{(1,13)}=6.74, p=0.02, \eta_{p}^{2}=0.34\right]$ and a significant interaction effect for the factors expertise and task $\left[F_{(1,13)}=8.92, p=0.01, \eta_{p}^{2}=0.41\right]$. Two separate Two-Way ANOVAs for the two tasks (chess/faces, with factors presentation and expertise) showed a significant main effect for expertise for chess related stimuli $\left[F_{(1,13)}=7.14, p=0.02, \eta_{p}^{2}=0.36\right]$ while no effect was observed in the control condition [faces, main effect expertise: $\left.F_{(1,13)}=0.86, p=0.37, \eta_{p}^{2}=0.06\right]$. In these analyses, there was no effect involving the factor presentation $(p>0.12)$.

\section{Left anterior TPJ}

In the anterior left TPJ ROI a similar result pattern emerged. There was a stronger activation in this region for experts than in novices depending on the stimulus material administered (Figure 3B). In this ROI experts also showed stronger activations for complex chess related stimuli, while no meaningful difference between experts and novices was observable for faces. This was approved by the statistical analysis: a Three-Way ANOVA showed a significant interaction effect for expertise and task $\left[F_{(1,13)}=\right.$ 15.09, $\left.p=0.002, \eta_{p}^{2}=0.54\right]$. The following separate ANOVAs for the two tasks revealed a significant main effect for expertise for chess stimuli $\left[F_{(1,13)}=7.50, p=0.017, \eta_{p}^{2}=0.37\right]$, while a significant main effect in the faces condition was present for the factor presentation only $\left[\mathrm{F}_{(1,13)}=6.67, p=0.02, \eta_{p}^{2}=0.34\right]$.

\section{Left posterior TPJ}

For the posterior left TPJ ROI the previous result pattern was not observable (see Figure 3C). The Three-Way ANOVA showed a significant main effect for presentation $\left[F_{(1,13)}=16.99, p=\right.$ $\left.0.001, \eta_{p}^{2}=0.57\right]$ and a significant interaction effect for the factors task and expertise $\left[F_{(1,13)}=8.68, p=0.011, \eta_{p}^{2}=0.40\right]$. In the subsequent Two-Way ANOVAs for the two different tasks (chess/faces) a significant main effect for presentation was observable in the chess task $\left[F_{(1,13)}=5.28, p=0.039, \eta_{p}^{2}=0.29\right]$ while no effect was present for faces $\left[F_{(1,13)}=2.84, p=0.12\right.$, $\left.\eta_{p}^{2}=0.18\right]$.

\section{EXPERIMENT 2}

For Experiment 2 a $2 \times 3$ repeated measures ANOVAs with the factors expertise (expert vs. novice) and task (check vs. identity vs. control) were calculated for each ROI. These analyses did not reveal any differences between experts and novices (see Figure 4).

\section{Right TPJ}

For the right TPJ region we found a significant main effect for task $\left[F_{(2,28)}=4.44, p=0.021, \eta_{p}^{2}=0.24\right]$. 


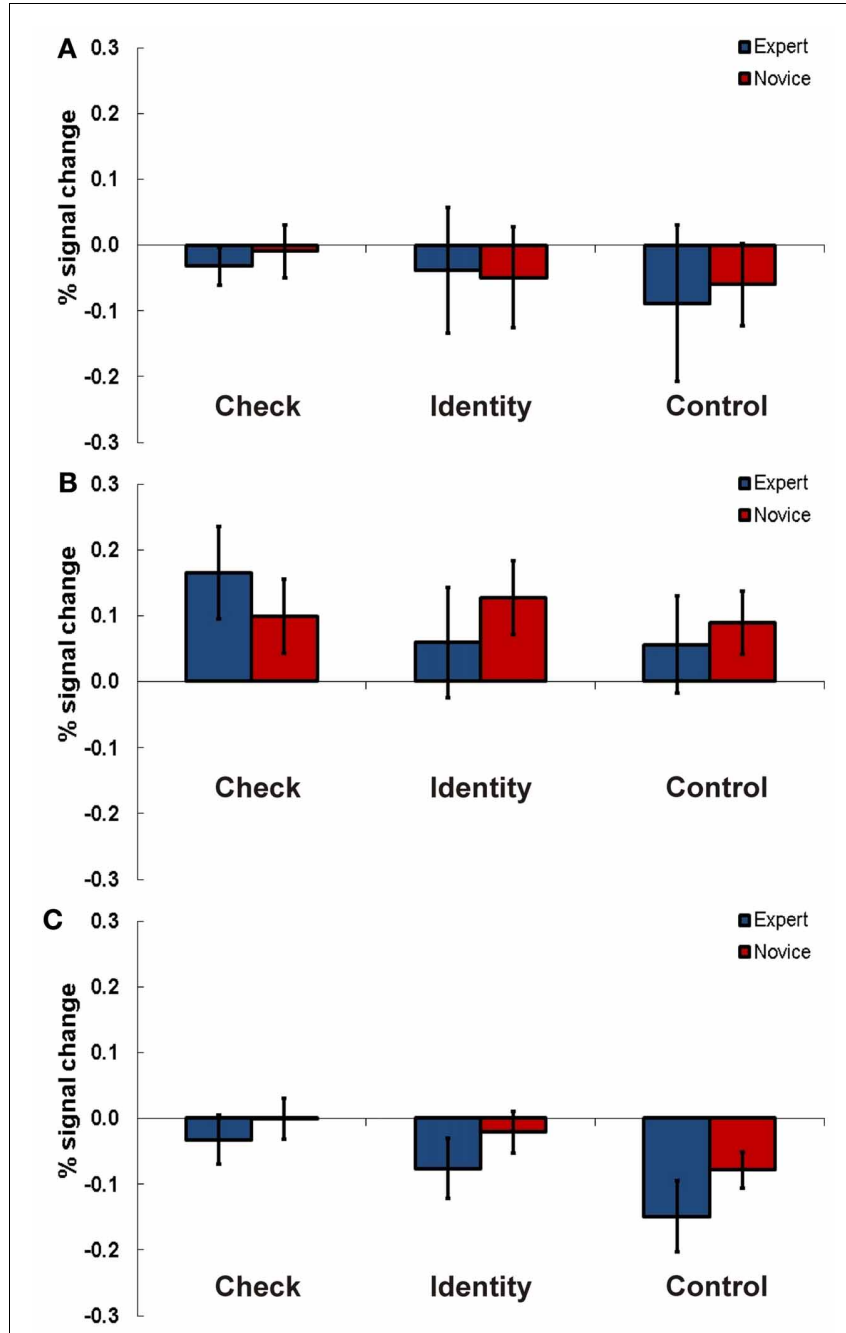

FIGURE 4 | Results Experiment 2. Percent signal change (PSC) for the three experimental conditions check (indicate if knight is in check), identity (recognition of a chess piece), and control (recognition of a geometrical shape) for experts and novices in TPJ ROI right (A), left anterior (B), and left posterior TPJ (C). Error bars indicate standard error of the mean.

\section{Left anterior TPJ}

Also in the anterior left TPJ area we found a significant main effect for task $\left[F_{(2,28)}=3.63, p=0.04, \eta_{p}^{2}=0.21\right]$. Additionally, the interaction of task and expertise was significant $\left[F_{(2,28)}=4.68\right.$, $\left.p=0.015, \eta_{p}^{2}=0.26\right]$. Post-hoc t-tests looking for significant differences between experts and novices in the three tasks did not show any significant results.

\section{Left posterior TPJ}

In the posterior left TPJ region the Two-Way ANOVA showed a significant main effect for task as well $\left[F_{(2,28)}=15.98\right.$, $\left.p=0.001, \eta_{p}^{2}=0.53\right]$.

\section{EXPERIMENT 3}

In this particular experiment a $2 \times 3 \times 2$ design was used. It contained the following factors and levels: expertise (expert vs.

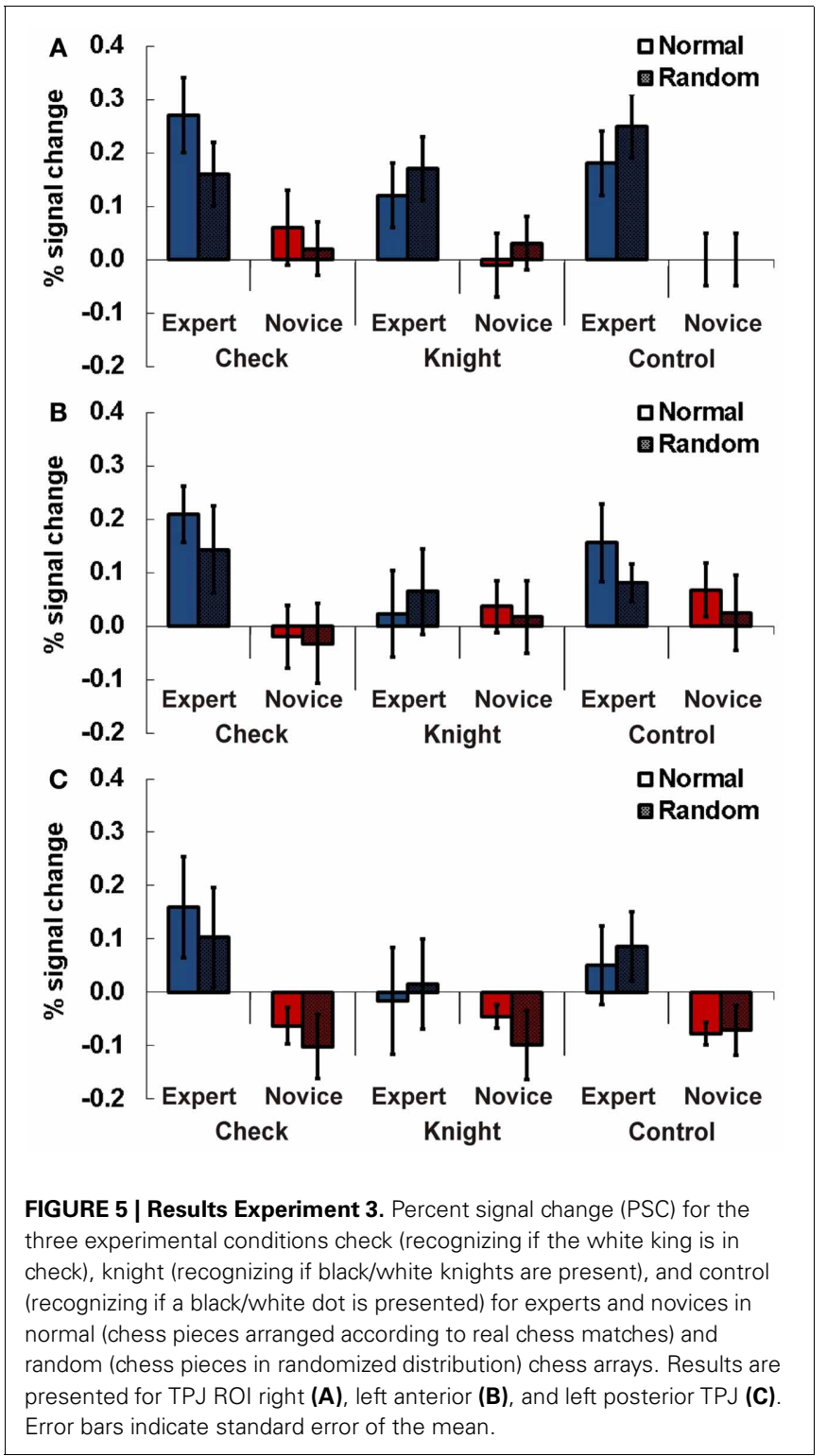

novice) $\mathrm{x}$ task (check vs. knight vs. dot) x position (normal vs. random).

\section{Right TPJ}

In the right-hemispheric TPJ region experts showed stronger activations compared to novices across all three tasks (see Figure 5A). A Three-Way ANOVA including all factors confirmed this observation by a significant main effect for expertise $\left[F_{(1,13)}=7.70\right.$, $\left.p=0.016, \eta_{p}^{2}=0.24\right]$. We observed a slightly non-significant interaction effect for the factors task and position $\left[F_{(1,13)}=3.82\right.$, $\left.p=0.07, \eta_{p}^{2}=0.19\right]$. No other main effects or interactions were significant (all $p>0.28)$.

\section{Left anterior TPJ}

In the anterior left-hemispheric ROI the Three-Way ANOVA revealed an interaction effect for the factors expertise and task just above the adopted type- 1 error probability threshold of 0.05 
$\left[F_{(2,26)}=3.12, p=0.06, \eta_{p}^{2}=0.20\right.$, see Figure 5B $]$. Subsequent separate $2 \times 2$ ANOVAs for the different tasks showed a significant main effect for expertise in the check task $\left[F_{(1,13)}=5.12\right.$, $\left.p=0.042, \eta_{p}^{2}=0.28\right]$.

\section{Left posterior TPJ}

The analysis for the posterior left-hemispheric ROI also revealed an interaction effect for the factors expertise and task slightly above the probability threshold $\left[F_{(2,26)}=3.23, p=0.056, \eta_{p}^{2}=\right.$ 0.20 , see Figure $5 \mathbf{C}$ ]. Separate $2 \times 2$ ANOVAs for the different tasks demonstrated a significant main effect for expertise in the check task $\left[F_{(1,13)}=4.78, p=0.048, \eta_{p}^{2}=0.27\right]$.

\section{EXPERIMENT 4}

For Experiment 4 a $2 \times 3 \times 2$ design was applied. It comprised the following factors and levels: expertise (expert vs. novice) $\times$ task (threat vs. knight $\&$ bishop vs. control) $\times$ position (normal vs. random).

\section{Right TPJ}

In the right-hemispheric TPJ region experts compared to novices showed higher signals for chess related stimuli than for control material (see Figure 6A). This result was confirmed by a ThreeWay ANOVA showing a significant main effect for expertise $\left[F_{(1,21)}=13.19, p=0.002, \eta_{p}^{2}=0.37\right]$ and an interaction effect for the factors expertise and task $\left[F_{(2,42)}=5.18, p=0.01, \eta_{p}^{2}=\right.$ 0.20]. In separate ANOVAs for every task (threat, knight \& bishop, control) significantly higher activations for complex chess stimuli were confirmed for chess experts compared to novices. The main effect for expertise was significant for the threat $\left[F_{(1,21)}=29.24\right.$, $\left.p<0.001, \eta_{p}^{2}=0.58\right]$ and the knight \& bishop task $\left[F_{(1,21)}=\right.$ 8.68, $\left.p=0.008, \eta_{p}^{2}=0.29\right]$, but slightly not for the control task $\left[F_{(1,21)}=3.65, p=0.07, \eta_{p}^{2}=0.15\right]$.

\section{Left anterior TPJ}

For the anterior left-hemispheric TPJ region we observed a similar result pattern. Experts compared to novices showed stronger neuronal activations for chess related stimuli than for control material (see Figure 6B). A Three-Way ANOVA confirmed this observation with a significant main effect for expertise $\left[F_{(1,21)}=12.42, p=0.002, \eta_{p}^{2}=0.40\right]$ and a significant interaction effect of expertise and task $\left[F_{(2,42)}=5.89, p=0.006\right.$, $\left.\eta_{p}^{2}=0.24\right]$. The subsequent Two-Way ANOVAs for the three tasks revealed significant main effects for the factor expertise for the threat $\left[F_{(1,21)}=5.54, p=0.029, \eta_{p}^{2}=0.23\right]$ and the knight \& bishop task $\left[F_{(1,21)}=10.20, p=0.005\right]$, while no effect was present for control material $\left[F_{(1,21)}=1.85, p=0.19\right.$, $\left.\eta_{p}^{2}=0.35\right]$.

\section{Left posterior TPJ}

In the posterior left TPJ we found a significant three-way interaction for expertise $\mathrm{x}$ task $\mathrm{x}$ position $\left[F_{(2,42)}=4.12\right.$, $p=0.024, \eta_{p}^{2}=0.18$, see Figure 6C]. Subsequent ANOVAs for every task showed a significant interaction for position and expertise $\left[F_{(1,21)}=4.39, p=0.05, \eta_{p}^{2}=0.19\right]$ in the knights \& bishop task. The following post-hoc $t$-tests supported a difference between experts and novices for normal $\left[T_{(21)}=2.32, p=0.03\right.$,

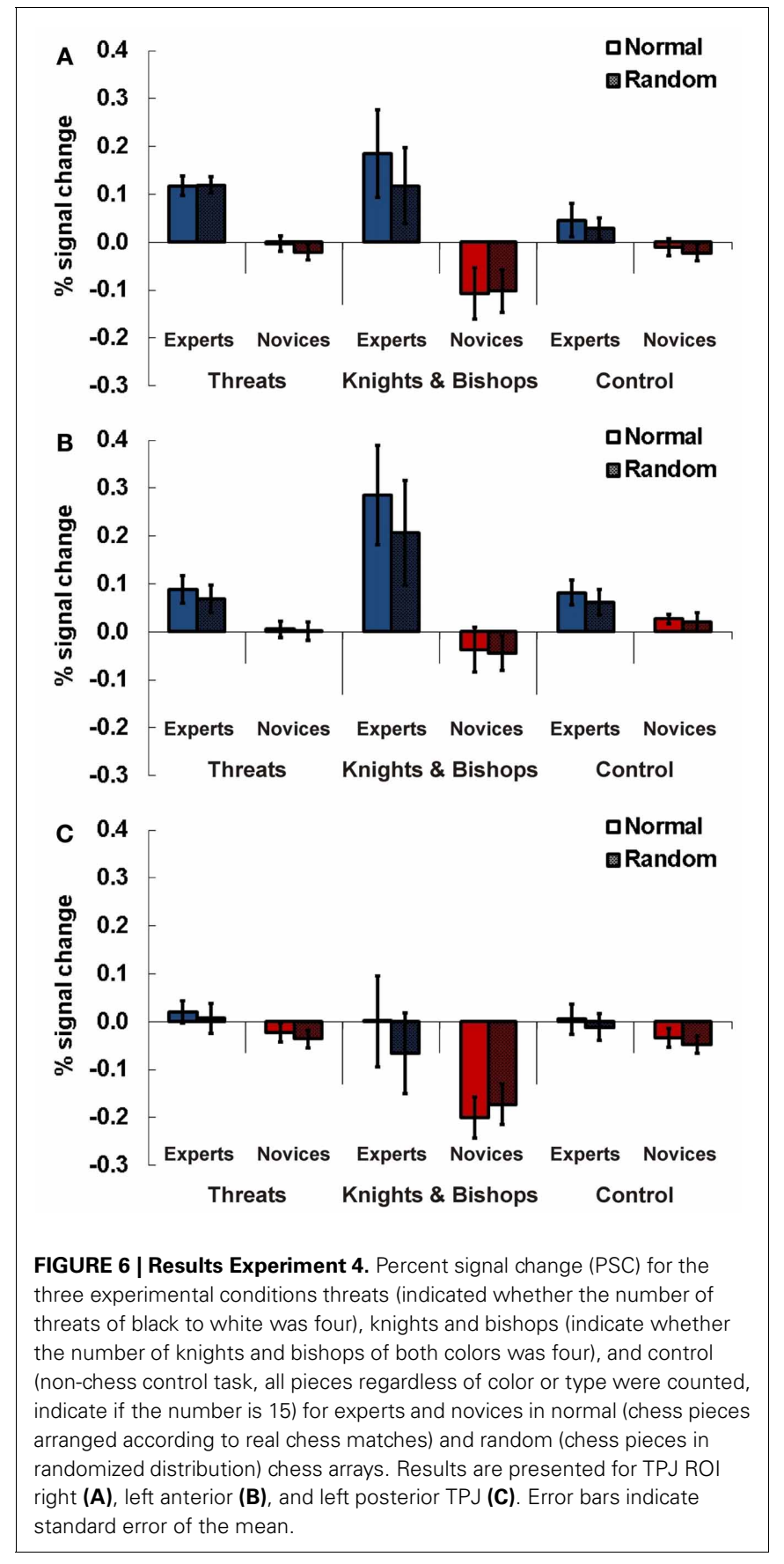

Cohen's $d=0.95]$ but not random presentations $\left[T_{(21)}=1.29\right.$, $p=0.21, d=0.59]$. However, the difference for normal presentations barely missed the priorily adopted significance threshold of $p=0.05$ after Bonferroni correction.

\section{DISCUSSION}

In a series of four independent ROI analyses the BOLD signal changes in bilateral TPJ areas during the perception of complex chess related visual stimuli were investigated. We examined possible neuronal differences between chess experts and novices in left and in right TPJ regions that were associated with localized 
signal increases in an independent experiment on global perception (Huberle and Karnath, 2012). We hypothesized that superior visual processing skills for highly familiar, complex material (expert view) are strongly associated with enhanced visual integration abilities: chess experts perceive chess situations rather at the global level (full chess board) whereas novices focus on the local level (individual chess pieces). Indeed, experts compared to novices showed higher signals in bilateral TPJ areas during the presentation of complex but highly familiar chess stimuli in three of four ROI analyses. These signal differences were consistent for all stimuli with meaningful, chess-related content. Furthermore, the absence of significant differences between experts and novices in experiment 2 is in good agreement with our hypothesis. The stimuli used in Experiment 2 (Bilalić et al., 2011a; see Figure 1C) displayed a simplified version of a checkerboard with three by three fields. In the other experiments stimuli consisted of full chess boards and multiple chess pieces in various configurations (Bilalić et al., 2010, 2011b, 2012; see Figures 1B,D,E).

Our observations strengthen previous data that suggested a significant role of the TPJ in the processing of complex object configurations (Huberle and Karnath, 2012). This assumption is in good agreement with our current knowledge about the typical bilateral area of damage or degeneration in patients with simultanagnosia (Rizzo and Hurtig, 1987; Friedman-Hill et al., 1995; Rafal, 1997; Karnath et al., 2000; Tang-Wai et al., 2004; Valenza et al., 2004; Huberle and Karnath, 2006; Huberle et al., 2010; Thomas et al., 2012). Against the assumption that TPJ plays a specific role for global perception it might be argued that it simply controls attentional switches between or the balancing of local and global visual inputs. Mosaic stimuli like the ones used by Huberle and Karnath (2012) would allow for a detection of global shapes by low scale visual feature detectors early in the visual system, balanced with information coming from high scale visual feature detectors by the TPJ. However, the observation of similar signal changes in a set of experiments using chess board stimuli argues against this interpretation. The relationships between the local items, i.e., chess pieces, are not created through physical features but through semantic relations between the local stimuli whereas the physical characteristics are substantially different from the typical stimuli used in studies on visual integration (e.g., Fink et al., 1996; Huberle and Karnath, 2012). Thus, we assume that our observation of consistent signal changes at the TPJ in two experimentally very different situations with substantially different stimulus material supports a role of TPJ in visual integration processes beyond attentional control.

The ROIs for the right and left hemisphere analyzed in the present study were different. Whereas a single ROI was analyzed for the right hemisphere, two separated ROIs were used for the left hemisphere. This was the consequence of the transfer of the functional definition of these ROIs from the preceding global perception experiment (Huberle and Karnath, 2012) to the chess expert datasets based on accepted voxel- and cluster-level thresholds. Thus, the definition of these regions was based on objective statistical criteria to allow reproducibility. Obviously, using other voxel- and cluster-level thresholds or slightly different first- and second-level statistics might have resulted in somewhat different delineations of the ROIs. However, the general pattern of the results would not differ. The signal patterns in the analyzed ROIs suggested a relative lateralization of visual integration to the right hemisphere. We found strong interaction effects including the factor expertise for the large right TPJ ROI, whereas only the anterior left TPJ ROI revealed consistent differences between experts and novices during the presentation of complex visual material across Experiments 1, 3, and 4. In contrast, the more posterior left TPJ region showed much less consistent results with a somewhat conclusive signal pattern only for Experiment 3. The idea of a relative lateralization that was not tested explicitly would be in agreement with several studies arguing for a right hemispheric specialization for global aspects in visual integration (Martin, 1979; Robertson et al., 1988; Fink et al., 1997b; Yamaguchi et al., 2000) and perception of complex chess configurations (Krawczyk et al., 2011). Nevertheless, one study reported a left hemispheric dominance for processing of global features of complex visual material (Fink et al., 1997b). This variability between fMRI studies depending on the particular task and samples may also indicate that global perception processes are bilaterally represented in the human left and right hemispheres. The fact that the vast majority of the patients showing simultanagnosia suffered bilateral brain damage (Rizzo and Hurtig, 1987; Friedman-Hill et al., 1995; Rafal, 1997; Karnath et al., 2000; Tang-Wai et al., 2004; Valenza et al., 2004; Huberle and Karnath, 2006, 2010; Thomas et al., 2012) supports this assumption.

The observed association of superior skills with an increased BOLD signal in a confined cortical structure is also in line with studies investigating the neuronal effects of visual perceptual training and expert view in other brain regions. However, in numerous functional imaging studies on perceptual learning it was demonstrated that training results in higher BOLD signals in task-related brain areas (Gauthier et al., 1999; Grill-Spector et al., 2000; Furmanski et al., 2004; Op de Beeck et al., 2006; Jastorff et al., 2009). Particularly in the context of global perception, an increase of BOLD signal amplitudes was associated with an improvement of complex stimulus processing through learning (Maertens and Pollmann, 2005; Zhang and Kourtzi, 2010; Zhang et al., 2010; Mayhew et al., 2012). Beyond, research on expert-novice differences showed higher BOLD signals in experts (Gauthier et al., 2000; Rhodes et al., 2004; Harley et al., 2009). Our observations may also be addressed to prolonged training effects causing modulation and fine-tuning of other non-visual areas (Moore et al., 2006; Guida et al., 2012). We therefore suggest that the increase of the BOLD signal in the TPJ represents an important contribution to the behavioral difference between experts and novices. Further, we did not observe any significant effects for inverted presentations or random chess positions, arguing for highly automatized global processing mechanisms for chess configurations in over-trained experts. Whereas other complex visual stimuli like faces become more or less incomprehensible by an inversion, chess boards are still interpretable. Therefore, we did not expect clear-cut inversion effects for global chess stimuli in the TPJ ROIs, similar to the well-established differences for faces in the respective brain areas (Epstein et al., 2006). 
However, neuroimaging studies of learning and expertise in other cognitive domains, like visual working memory (WM), showed different or even opposite BOLD result patterns with behavioral changes (Landau et al., 2004; Kelly and Garavan, 2005; Jaeggi et al., 2007). Jaeggi et al. (2007) demonstrated higher BOLD signals in low-performers than in experts in a working memory task. Landau and colleagues (Landau et al., 2004) found that learning led to a decrease of BOLD signals in several cortical brain areas. Obviously, there may exist several other factors, like advantages in working memory or motivation driving neuronal signals in expert view. However, the present study highlights an important contribution of visual integration and the associated neuronal structures to superior visual skills in chess experts.

In conclusion, our data show that fMRI signals in the TPJ are increased during the observation of complex stimuli in experts who experienced an extensive training that most likely resulted in superior skills of visual integration. The results of

\section{REFERENCES}

Bálint, R. (1909). Seelenlähmung des "Schauens," optische Ataxie, räumliche Störung der Aufmerksamkeit. Monatsschr. Psychiatr. Neurol. 25, 51-81.

Bilalić, M., Kiesel, A., Pohl, C., Erb, M., and Grodd, W. (2011a). It takes two-skilled recognition of objects engages lateral areas in both hemispheres. PLOS ONE 6:e16202. doi: 10.1371/journal.pone.0016202

Bilalić, M., Langner, R., Ulrich, R., and Grodd, W. (2011b). Many faces of expertis33 fusiform face area in chess experts and novices. J. Neurosci. 31, 10206-10214. doi: 10.1523/JNEUROSCI.5727-10.2011

Bilalić, M., Langner, R., Erb, M., and Grodd, W. (2010). Mechanisms and neural basis of object and pattern recognition: a study with chess experts. J. Exp. Psychol. Gen. 139, 728-742. doi: 10.1037/a0020756

Bilalić, M., McLeod, P., and Gobet, F. (2008a). Expert and "novice" problem solving strategies in chess: sixty years of citing de Groot, (1946). Think. Reason. 14, 395-408. doi: 10.1080/13546780802265547

Bilalić, M., McLeod, P., and Gobet, F. (2008b). Inflexibility of expertsreality or myth? Quantifying the Einstellung effect in chess masters. Cogn. Psychol. 56, 73-102. doi: 10.1016/j.cogpsych.2007.02.001

Bilalić, M., McLeod, P., and Gobet, F. (2009). Specialization effect and its influence on memory and problem solving in expert chess players. Cogn. Sci. 33, 1117-1143. doi: 10.1111/j.1551-6709.2009.01030.x

Bilalić, M., McLeod, P., and Gobet, F. (2010). The mechanism of the Einstellung (Set) Effect: a pervasive source of cognitive bias. Curr.
Dir. Psychol. Sci. 19, 111-115. doi: 10.1177/0963721410363571

Bilalić, M., Turella, L., Campitelli, G., Erb, M., and Grodd, W. (2012). Expertise modulates the neural basis of context dependent recognition of objects and their relations. Hum. Brain Mapp. 33, 2728-2740. doi: 10.1002/hbm.21396

Chase, W. G., and Simon, H. A. (1973). Perception in chess. Cogn. Psychol. 4, 55-81. doi: 10.1016/0010-0285(73) 90004-2

DeGroot, A. D. (1978). Thought and Choice in Chess. New York, NY: Mouton De Gruyter.

Elo, A. E. (1978). The Rating of Chess Players, Past and Present. New York, NY: Arco.

Epstein, R. A., Higgins, J. S., Parker, W., Aguirre, G. K., and Cooperman, S. (2006). Cortical correlates of face and scene inversion: a comparison. Neuropsychologia 44, 1145-1158. doi: $\quad 10.1016 /$ j.neuropsychologia. 2005.10.009

Ericsson, K. A., Krampe, R. T., and Tesch-Römer, C. (1993). The role of deliberate practice in the acquisition of expert performance. Psychol. Rev. 100, 363-406. doi: 10.1037/0033295X.100.3.363

Fink, G. R., Halligan, P. W., Marshall, J. C., Frith, C. D., Frackowiak, R. S., and Dolan, R. J. (1996). Where in the brain does visual attention select the forest and the trees? Nature 382, 626-628. doi: 10.1038/382626a0

Fink, G. R., Halligan, P. W., Marshall, J. C., Frith, C. D., Frackowiak, R. S., and Dolan, R. J. (1997a). Neural mechanisms involved in the processing of global and local aspects of hierarchically organized visual stimuli. Brain 120(Pt 1), 1779-1791.

our cross-paradigm ROI analyses shows that such signal increases are not only observed using highly selective global/local stimulus material in within-subject comparisons but can be detected in between-subject comparisons using stimulus material from a different field of research. In good agreement with previous fMRI studies (Himmelbach et al., 2009; Huberle and Karnath, 2012) and patient reports (Rizzo and Hurtig, 1987; FriedmanHill et al., 1995; Rafal, 1997; Karnath et al., 2000; Tang-Wai et al., 2004; Valenza et al., 2004; Huberle and Karnath, 2006; Huberle et al., 2010; Thomas et al., 2012) the presented data supports the assumption of a crucial involvement of the left and the right TPJ in global gestalt perception.

\section{ACKNOWLEDGMENTS}

This work was supported by the European Union (ERC StG 211078), the DFG (Ka 1258/10-1; Bi 1450/1-2) and the Open Access Publishing Fund of the University of Tübingen.

Fink, G. R., Marshall, J. C., Halligan, P. W., Frith, C. D., Frackowiak, R. S., and Dolan, R. J. (1997b). Hemispheric specialization for global and local processing: the effect of stimulus category. Proc. Biol. Sci. 264, 487-494. doi: 10.1098/rspb.1997.0070

Friedman-Hill, S. R., Robertson, L. C., and Treisman, A. (1995). Parietal contributions to visual feature binding: evidence from a patient with bilateral lesions Science 269, 853-855. doi 10.1126/science.7638604

Furmanski, C. S., Schluppeck, D., and Engel, S. A. (2004). Learning strengthens the response of primary visual cortex to simple patterns. Curr. Biol. 14, 573-578. doi 10.1016/j.cub.2004.03.032

Gauthier, I., Skudlarski, P., Gore, J. C., and Anderson, A. W. (2000). Expertise for cars and birds recruits brain areas involved in face recognition. Nat. Neurosci. 3, 191-197. doi: $10.1038 / 72140$

Gauthier, I., Tarr, M. J., Anderson, A. W., Skudlarski, P., and Gore, J. C. (1999). Activation of the middle fusiform "face area" increases with expertise in recognizing novel objects. Nat. Neurosci. 2, 568-573. doi: $10.1038 / 9224$

Gobet, F., and Simon, H. A. (1996). Templates in chess memory: a mechanism for recalling several boards. Cogn. Psychol. 31, 1-40. doi: 10.1006/cogp.1996.0011

Gobet, F., and Waters, A. J. (2003) The role of constraints in expert memory. J. Exp. Psychol. Learn. Mem. Cogn. 29, 1082-1094. doi: 10.1037/0278-7393.29.6.1082

Goebel, R., Esposito, F., and Formisano, E. (2006). Analysis of functional image analysis contest (FIAC) data with brainvoyager QX: from single-subject to cortically aligned group general linear model analysis and self-organizing group independent component analysis. Hum Brain Mapp. 27, 392-401. doi: 10.1002/hbm.20249

Grill-Spector, K., Kushnir, T., Hendler, T., and Malach, R. (2000). The dynamics of object-selective activation correlate with recognition performance in humans. Nat. Neurosci. 3, 837-843. doi: 10.1038/77754

Guida, A., Gobet, F., Tardieu, H., and Nicolas, S. (2012). How chunks, long-term working memory and templates offer a cognitive explanation for neuroimaging data on expertise acquisition: a two-stage framework. Brain Cogn. 79, 221-244. doi: 10.1016/j.bandc.2012.01.010

Harley, E. M., Pope, W. B., Villablanca, J. P., Mumford, J., Suh, R., Mazziotta, J. C., et al. (2009). Engagement of fusiform cortex and disengagement of lateral occipital cortex in the acquisition of radiological expertise. Cereb. Cortex 19, 2746-2754. doi: 10.1093/cercor/bhp051

Himmelbach, M., Erb, M., Klockgether, T., Moskau, S., and Karnath, H. O. (2009). fMRI of global visual perception in simultanagnosia. Neuropsychologia 47, 1173-1177. doi: $\quad 10.1016 /$ j.neuropsychologia. 2008.10.025

Huberle, E., Driver, J., and Karnath, H.-O. (2010). Retinal versus physical stimulus size as determinants of visual perception in simultanagnosia. Neuropsychologia 48, 1677-1682. doi: 10.1016/ j.neuropsychologia.2010.02.013 
Huberle, E., and Karnath, H.-O. (2006). Global shape recognition is modulated by the spatial distance of local elementsevidence from simultanagnosia. Neuropsychologia 44, 905-911. doi: $\quad 10.1016 /$ j.neuropsychologia. 2005.08.013

Huberle, E., and Karnath, H.-O. (2010). Saliency modulates global perception in simultanagnosia. Exp. Brain Res. 204, 595-603. doi: 10.1007/s00221-010-2328-x

Huberle, E., and Karnath, H.-O. (2012). The role of temporo-parietal junction (TPJ) in global Gestalt perception. Brain Struct. Funct. 217, 735-746. doi: 10.1007/s00429-0110369-y

Jaeggi, S. M., Buschkuehl, M., Etienne, A., Ozdoba, C., Perrig, W. J., and Nirkko, A. C. (2007). On how high performers keep cool brains in situations of cognitive overload. Cogn. Affect. Behav. Neurosci. 7, 75-89. doi: 10.3758/CABN.7.2.75

Jastorff, J., Kourtzi, Z., and Giese, M. A. (2009). Visual learning shapes the processing of complex movement stimuli in the human brain. J. Neurosci. 29, 14026-14038. doi: $10.1523 /$ JNEUROSCI.307009.2009

Karnath, H., Ferber, S., Rorden, C., and Driver, J. (2000). The fate of global information in dorsal simultanagnosia. Neurocase 6, 295-306. doi: $10.1080 / 13554790008402778$

Kelly, A. M. C., and Garavan, H. (2005). Human functional neuroimaging of brain changes associated with practice. Cereb. Cortex 15, 1089-1102. doi: 10.1093/cercor/bhi005

Koffka, K. (1935). Principles of Gestalt Psychology. 1st Edn. New York, NY: Harcourt.

Krawczyk, D. C., Boggan, A. L., McClelland, M. M., and Bartlett, J. C. (2011). The neural organization of perception in chess experts. Neurosci. Lett. 499, 64-69. doi: 10.1016/j.neulet.2011.05.033

Lancaster, J. L., Tordesillas-Gutiérrez, D., Martinez, M., Salinas, F., Evans, A., Zilles, K., et al. (2007). Bias between MNI and Talairach coordinates analyzed using the ICBM-152 brain template. Hum.
Brain Mapp. 28, 1194-1205. doi: 10.1002/hbm.20345

Landau, S. M., Schumacher, E. H., Garavan, H., Druzgal, T. J., and D'Esposito, M. (2004). A functional MRI study of the influence of practice on component processes of working memory. Neuroimage 22, 211-221. doi: 10.1016/j.neuroimage.2004.01.003

Leube, D. T., Erb, M., Grodd, W. Bartels, M., and Kircher, T. T. (2001). Differential activation in parahippocampal and prefrontal cortex during word and face encoding tasks. Neuroreport 12, 2773-2777. doi: 10.1097/00001756200108280-00035

Leube, D. T., Erb, M., Grodd, W., Bartels, M., and Kircher, T. T. J. (2003). Successful episodic memory retrieval of newly learned faces activates a left frontoparietal network. Brain. Res. Cogn. Brain Res. 18, 97-101. doi: 10.1016/j.cogbrainres.2003.09.008

Maertens, M., and Pollmann, S. (2005). fMRI reveals a common neural substrate of illusory and real contours in V1 after perceptual learning. $J$. Cogn. Neurosci. 17, 1553-1564. doi: 10.1162/089892905774597209

Martin, M. (1979). Hemispheric specialization for local and global processing. Neuropsychologia 17, 33-40. doi: 10.1016/0028-3932(79)90019-8

Mayhew, S. D., Li, S., and Kourtzi, Z. (2012). Learning acts on distinct processes for visual form perception in the human brain J. Neurosci. 32, 775-786. doi: 10.1523/JNEUROSCI.2033-11.2012

Moore, C. D., Cohen, M. X., and Ranganath, C. (2006). Neural mechanisms of expert skills in visual working memory. $J$. Neurosci. 26, 11187-11196. doi: 10.1523/JNEUROSCI.1873-06.2006

Navon, D. (1977). Forest before trees: the precedence of global features in visual perception. Cogn. Psychol. 9, 353-383. doi: 10.1016/0010-0285 (77) $90012-3$

Op de Beeck, H. P., Baker, C. I., DiCarlo, J. J., and Kanwisher, N. G. (2006). Discrimination training alters object representations in human extrastriate cortex. $J$.
Neurosci. 26, 13025-13036. doi: 10.1523/JNEUROSCI.2481-06.2006

Rafal, R. (1997). "Balint syndrome," in Behavioral Neurology and Neuropsychology, eds T. Feinberg and M. Farah (Boston, MA: McGraw-Hill), 337-356.

Rhodes, G., Byatt, G., Michie, P. T., and Puce, A. (2004). Is the fusiform face area specialized for faces, individuation, or expert individuation? J. Cogn. Neurosci. 16, 189-203. doi $10.1162 / 089892904322984508$

Rizzo, M., and Hurtig, R. (1987) Looking but not seeing: attention, perception, and eye movements in simultanagnosia. Neurology 37, 1642-1648. doi: 10.1212/WNL.37.10.1642

Robertson, L. C., Lamb, M. R., and Knight, R. T. (1988). Effects of lesions of temporal-parietal junction on perceptual and attentional processing in humans. J. Neurosci. 8 , 3757-3769.

Tang-Wai, D. F., Graff-Radford, N. R., Boeve, B. F., Dickson, D. W., Parisi, J. E., Crook, R., et al. (2004). Clinical, genetic, and neuropathologic characteristics of posterior cortical atrophy. Neurology 63, 1168-1174 doi: 10.1212/01.WNL.0000140289. 18472.15

Thomas, C., Kveraga, K., Huberle, E., Karnath, H.-O., and Bar, M. (2012). Enabling global processing in simultanagnosia by psychophysical biasing of visual pathways. Brain 135, 1578-1585. doi: 10.1093/brain/ aws066

Valenza, N., Murray, M. M., Ptak, R., and Vuilleumier, P. (2004). The space of senses: impaired crossmodal interactions in a patient with Balint syndrome after bilateral parietal damage. Neuropsychologia 42, 1737-1748. doi: 10.1016/ j.neuropsychologia.2004.05.001

Vicente, K. J., and Wang, J. H. (1998). An ecological theory of expertise effects in memory recall. Psychol. Rev. 105, 33-57. doi: 10.1037/0033295X.105.1.33

Wertheimer, M. (1923) Untersuchungen zur Lehre von der Gestalt. Psychol. Forsch. 4, 301-350. doi: 10.1007/BF00410640
Yamaguchi, S., Yamagata, S., and Kobayashi, S. (2000). Cerebral asymmetry of the "top-down" allocation of attention to global and local features. J. Neurosci. 20, RC72.

Zaretskaya, N., Anstis, S., and Bartels, A. (2013). Parietal cortex mediates conscious perception of illusory gestalt. J. Neurosci. 33, 523-531. doi: 10.1523/ JNEUROSCI.2905-12.2013

Zhang, J., and Kourtzi, Z. (2010). Learning-dependent plasticity with and without training in the human brain. Proc. Natl. Acad. Sci. U.S.A. 107, 13503-13508. doi: 10.1073/pnas.1002506107

Zhang, J., Meeson, A., Welchman, A. E., and Kourtzi, Z. (2010). Learning alters the tuning of functional magnetic resonance imaging patterns for visual forms. J. Neurosci. 30, 14127-14133. doi: 10.1523/JNEUROSCI.2204-10.2010

Conflict of Interest Statement: The authors declare that the research was conducted in the absence of any commercial or financial relationships that could be construed as a potential conflict of interest.

Received: 29 April 2013; accepted: 10 August 2013; published online: 28 August 2013.

Citation: Rennig J, Bilalić M, Huberle $E$, Karnath $\mathrm{H}-\mathrm{O}$ and Himmelbach $M$ (2013) The temporo-parietal junction contributes to global gestalt perceptionevidence from studies in chess experts. Front. Hum. Neurosci. 7:513. doi: 10.3389/fnhum.2013.00513

This article was submitted to the journal Frontiers in Human Neuroscience. Copyright () 2013 Rennig, Bilalić, Huberle, Karnath and Himmelbach. This is an open-access article distributed under the terms of the Creative Commons Attribution License (CC BY). The use, distribution or reproduction in other forums is permitted, provided the original author(s) or licensor are credited and that the original publication in this journal is cited, in accordance with accepted academic practice. No use, distribution or reproduction is permitted which does not comply with these terms. 